\title{
Quantum Well Intermixing for Photonic IC Applications
}

\author{
E. Herbert $\mathrm{Li}^{\dagger}$ \\ University of Waterloo \\ Department of Electrical and Computer Engineering \\ Waterloo, Ontario, Canada
}

\begin{abstract}
Photonics IC is an attractive information processing means to fully utilize the enormous bandwidth carried by the optical signals. The full integration of photonics devices, such as tunable lasers, modulators and photodetectors have to be developed and which can be obtain by using the Quantum Well Intermixing technology. This paper will explore on the wavelength tunability using different Quantum Well Intermixing techniques, such as impurity induced diffusion and impurity-free vacancy diffusion. Emphasis will be put on the development of the very recent innovations and applications.
\end{abstract}

Key words: Quantum Well Intermixing, Thermal Interdiffusion, Optical Properties of Quantum Well, Optical Devices, Optoelectronic Devices.

\section{INTROUDUCTION}

A practical technique for integrating active and passive photonic devices will produce an immense impact on information processing, distribution, and manipulation. It is expected to be as significant a technological advance as was the development of the electronic IC. However, despite substantial efforts made by a large number of groups worldwide over the last few years, and the increasing demand for multiwavelength optical networks generated by the exponential growth of the internet and the World Wide Web in particular, this utopian vision has yet to be realized, where a consequence of the magnitude of the technological challenges is involved. For practical photonic IC's, practicality mandates low manufacturing cost and high reliability (low operating cost) even at the expense of somewhat reduced performance. With this in mind, we can establish the most important requirement for monolithically integrating optoelectronic devices of differing functionality's in order to achieve practical waveguiding photonic IC's. One of the primary requirements for this waveguiding is bandgap (operation wavelength) compatibility among the various optoelectronic devices. Although a number of techniques exist, each has its advantages and disadvantages - none is a panacea.

This talk presents a simple technique of fabricating integrated optoelectronic devices using spatially selective modification of quantum well $(\mathrm{QW})$ shape after standard growth which, in turn, modifies $\mathrm{QW}$ bandgap energy. This diffused quantum well (DFQW) is a non-square quantum well produced by interdiffusion of constituent atoms through the heterointerface (Fig. 1). In the literature, $\mathrm{DFQW}$ is also referred to as quantum well mixing or intermixing (QWI) and quantum well disordering [1]. It is based on the fact that a QW is an inherently metastable system due to the large concentration gradient of atomic species across the well/barrier interface. For example, in InGaAs/InGaAsP QWs, the phosphorous concentration changes from $43 \%$ to $0 \%$ in a distance of less than $1 \mathrm{~nm}$. At temperature above $750^{\circ} \mathrm{C}$ significant diffusion of atomic species will occur resulting in an interdiffusion of the well and barrier alloys. This process causes an increase in the bandgap energy. It can also be greatly enhanced by the presence of impurities or defects or vacancies in the vicinity of the interfaces of the QW, allowing interdiffusion to occur at temperatures that are substantially lower than that normally required. All these techniques are spatially selective, permitting interdiffusion enhancement only in the regions requiring a large bandgap, leaving other regions unmodified.

\footnotetext{
${ }^{\dagger}$ Permanent Address : University of Hong Kong, Department of Electrical and Electronic Engineering, Pokfulam Road, Hong Kong. Email : ehli@eee.hku.hk
} 
(a)

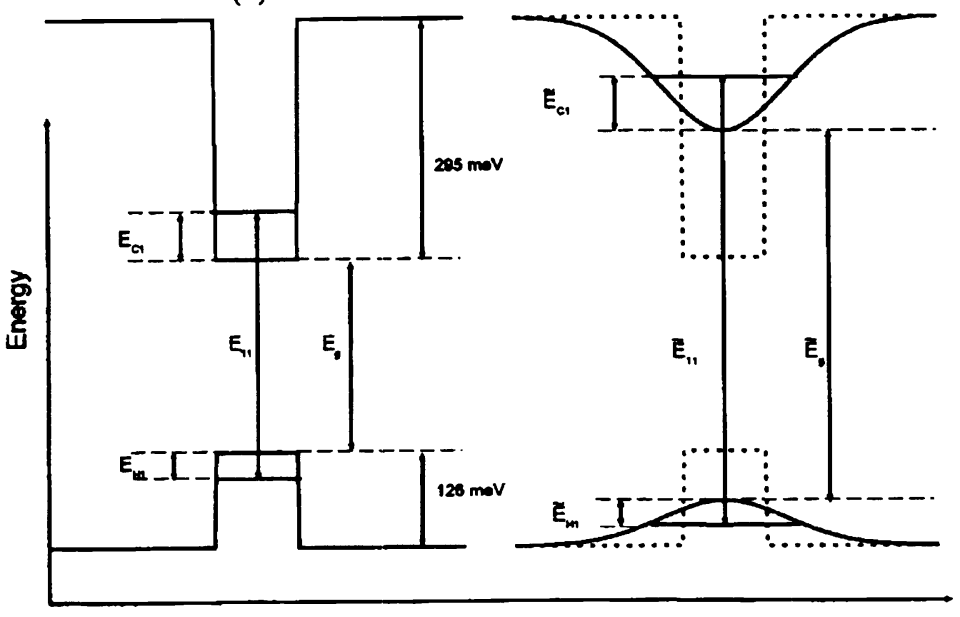

z (growth direction)

Figure 1. The conduction band and valence band potential energy profiles for an as-grown rectangular SQW structure and that of its interdiffused structure where $\mathrm{L}_{d} \approx 1 / 2 \mathrm{~L}_{\mathrm{z}}$. The tilde denotes the respective parameter in an interdiffused SQW and $\mathrm{E}_{\mathrm{cf}}$ is the sum of the electron $E_{\mathrm{c} 1}$ and hole $\mathrm{E}_{\mathrm{h} 1}$ confinement energies.

This bandgap modification is a powerful technique for monolithically integrating optoelectronic devices of varying functionalities on a single wafer. It is simple, has no deleterious effects on the optical, electrical, or lifetime properties of optoelectronic devices and, in fact, possesses surprising advantages (reducing polarization sensitivity and enhancing reflectance change). Since the process is easy to implement, it will simplify the fabrication of many complex photonic IC's and may finally render them practical enough to be adopted by industry. The advantage this technology is, through interdiffusion (intermixing), to accruate modify the QW materials ( $\mathrm{AlGaAs} / \mathrm{GaAs}$, InGaAs/GaAs and $\mathrm{InGaAs}(\mathrm{P}) / \mathrm{InP})$ bandgap and optical properties, and to make use of these tuning ability to integrate several device structures (such as waveguide-detector, laser-modulator, and laserlaser) to form photonic IC's. In particular, this allows a multi-section integrated structure will be fabricated for wide band-width and multi-wavelengths applications, such as to demonstrate its use for wavelength division multiplexing (WDM) in high bit rate communication systems.

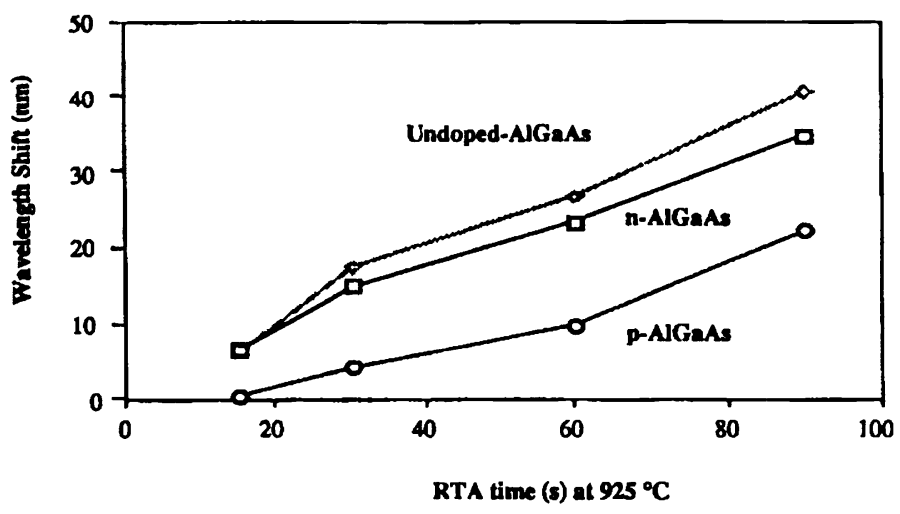

Figure 2. Wavelength shifts as a function of annealing time obtained from $\mathrm{SiO}_{2}$ capped intrinsic, n-I-o, and p-I-n samples annealed at $925^{\circ} \mathrm{C}$. 
Impurity-free vacancy disordering using $\mathrm{SiO}_{2}$ and $\mathrm{SrF}_{2}$ dielectric caps (Fig. 2) to induce selective quantum-well (QW) intermixing in the GaAs-AIGaAs system has also been developed. The intermixing rate of IFVD was found to be higher in $n-i-p$ and intrinsic than in p-i-n structures, which suggests that the diffusion of the Group III vacancy is not supported in p-type material. Single-mode waveguides have been fabricated from both as-grown and bandgap-tuned double-quantum-well (DQW) laser samples. Propagation losses as low as $8.5 \mathrm{~dB} \mathrm{~cm}^{-1}$ were measured from the bandgap-tuned waveguides at the lasing wavelength of the undisordered material, i.e., $860 \mathrm{~nm}$. Simulation was also carried out to study the contribution of free-carrier absorption from the cladding layers, and the leakage loss induced by the heavily p-doped GaAs contact layer. It was found that the leakage loss contributed by the GaAs cap layer is significant and increases with wavelength. Based on IFVD, the fabrication of multiple-wavelength lasers and multichannel wavelength division multiplexers were also demonstrated using the one-step "selective intermixing in selected area" technique. This technique enables one to control the degree of intermixing across a wafer. Lasers with bandgaps tuned to five different positions have been fabricated on a single chip (Fig. 3). These lasers showed only small changes in transparency current, internal quantum efficiency, or internal propagation loss, which indicates that the quality of the material remains high after being intermixed. Four-channel wavelength demultiplexers based on a waveguide photodetector design have also been fabricated (Fig. 4). Photocurrent and spontaneous emission spectra from individual diodes showed that the absorption edge was shifted by different degrees due to the selective degree of QW intermixing. The results obtained also imply that the technique can be used in the fabrication of broad-wavelength emission superluminenscent diodes.

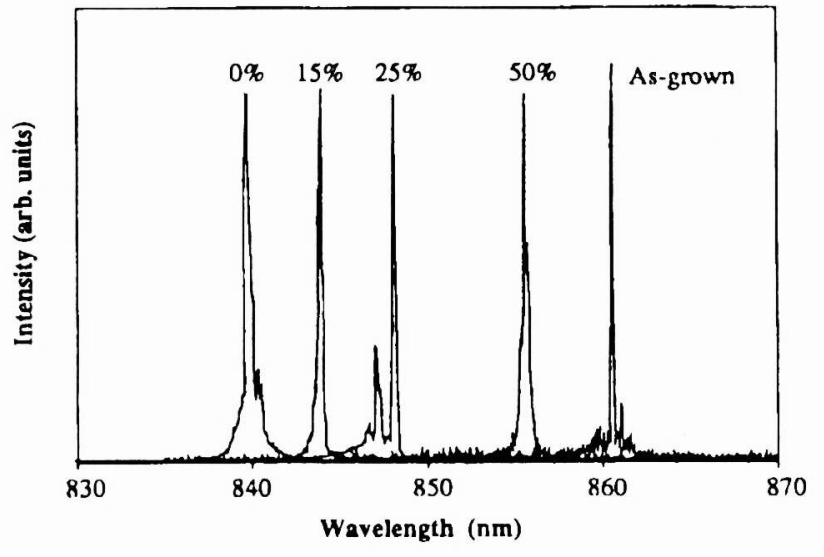

Figure 3. The lasing spectra of lasers bandgap-shifted using the SISA technique.

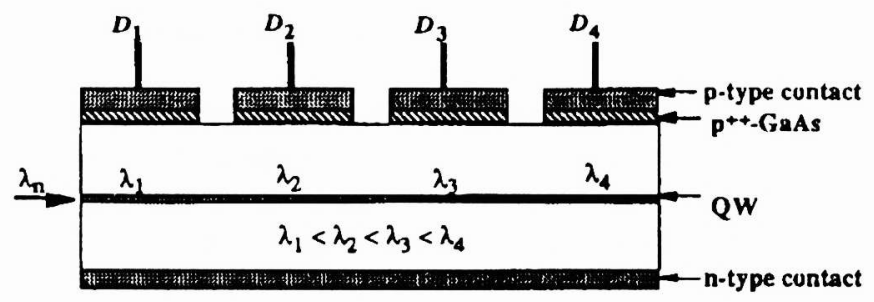

Figure 4. Schematic cross section of the four-channel demultiplexing wavefuide photodetector. The length of each diode is $300 \mathrm{~m}$ and the spacing between diodes is $100 \mathrm{~m}$. Light was injected from the cleaved edge of diode $\mathrm{D}_{1}$ during the photocurrent measurement.

During the past six months, much progress has been made regarding the use of diffused QW as a tool [3-11]. More precisely, a number of new techniques have been adopted to serve the purpose of QW intermixing, namely anodic-oxideinduced intermixing $[7,8]$, laser-assisted disordering $[9,10]$ and proton irradiation [11]. As for the diffusion mechanism [12, 13], computer simulations of double crystal x-ray diffraction (DCXRD) curves for annealed multiple QW structure have been used. In addition, much work has been done on the modified optical properties as a result of intermixing and its application to enhancing the device performance [14-17]. As regards the devices employed, more effort has been put into refining their applications, resulting in more sophisticated use of such devices [18-28]. In particular, the utilization of vertical cavity devices in the production of waveguides is a relatively novel attempt.

The latest progress and development in DFQWs will be summarized in the following sections. 


\section{TECHNIQUES FOR QUANTUM WELL INTERMIXING}

2.1 Ion Implantation

Ion Implantation is a technique in which direct injection of ionized, energetic atoms or molecules into a solid is employed. The ions injected will carry energies ranging from a few $\mathrm{keV}$ to several $\mathrm{MeV}$, and implant doses from $10^{10}$ to more than $10^{16} \mathrm{ions} / \mathrm{cm}^{2}$. Using small implantation energy, the damage introduced to the lattice structure will be reduced, but with a decrease in the penetration depth; whereas with small dose of implants, there will be less enhancement of diffusion rate.

Quantum well intermixing using high-energy ion implantation is a promising technique for laterally selective, postgrowth modification of a quantum well structure [3]. The lateral selectivity of the technique is a function of the ion straggling during implantation and the lateral diffusion of defects during post-implantation annealing, see Fig. 5. Low temperature photoluminescence was used to monitor the intermixng of quantum wells. A significant amount of intermixing, which resulted in a $57 \mathrm{~nm}$ blueshift of the QW bandgap energy, was observed when the mask stripe width was less than $5 \mu \mathrm{m}$, thus giving a lateral selectivity of $2.5 \mu \mathrm{m}$.

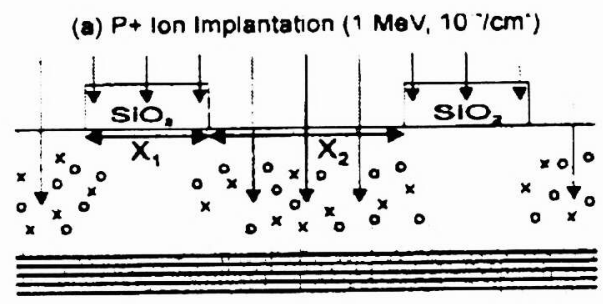

(b) after Rapid Thermal Anneal $\left(675^{\circ} \mathrm{C}\right)$

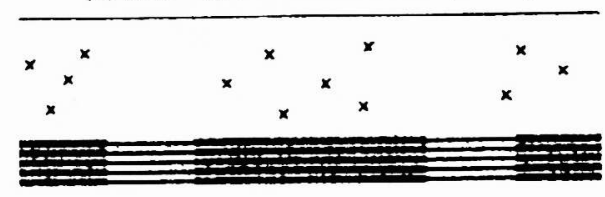

(c) Lateral Profile of Bandgap

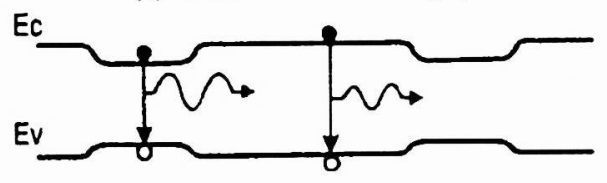

Figure 5. Schematic cross section through a sample illustrating selective area intermixing of five quantum wells located 1.77 um below the surface: (a) ion implantation of $\mathrm{P}^{+}$ions using a patterned $2.0 \mathrm{um}$ thick silicon dioxide mask; (b) after rapid thermal annealing, QW's underneath implanted regions and near the edges of masked regions have been intermixed; (c) the resultant change in the band-gap energy of the QW's along the lateral direction.

Using As implantation at $45 \mathrm{keV}$, the fabrication of $\mathrm{GaAs} / \mathrm{AlGaAs} \mathrm{QW}$ wires has been performed [2]. Photoreflectance measurements showed that wires appeared to have a parabolic lateral potential, and clear interwire coupling was observed from samples with barriers narrower than $50 \mathrm{~nm}$. The intermixing process was characterized by the giving of optimized annealing 
parameters of $875^{\circ} \mathrm{C}$ for $30 \mathrm{sec}$ and an implantation dose of $1 \times 10^{13} \mathrm{~cm}^{-2}$. Wire widths ranging between 35 and $1000 \mathrm{~nm}$, with a similar interwire spacing, were fabrication using e-beam lithography and a lift-off process. Broad PL spectra from 1000 to $150 \mathrm{~nm}$ with an obvious wavelength peak at about $750 \mathrm{~nm}$ was observed and a shoulder estimated to appear at about $733 \mathrm{~nm}$, indicating a lateral potential height of $38 \mathrm{meV}$. The lateral well indicated significantly small shifts for wire widths between 1000 and $150 \mathrm{~nm}$. Below $150 \mathrm{~nm}$, the wire emission began to show a significant blueshift and eventually merges with the lateral barrier for samples with $35 \mathrm{~nm}$ wires. Judging from these results, an intermixing radius of about $17 \mathrm{~nm}$ was estimated.

\subsection{Impurity Free Vacancy Diffusion}

The mechanism of IFVD requires the encapsulation of MQW samples by a dielectric cap such as $\mathrm{SiO}_{2}$ or $\mathrm{Si}_{3} \mathrm{~N}_{4}$ and then annealing at high temperature around $850{ }^{\circ} \mathrm{C} \sim 900^{\circ} \mathrm{C}$ for 30 to 200 seconds. This will lead to out-diffusion of Ga into the cap and vacancies are generated on the group III sublattice that diffuse to the barriers and promote the interdiffusion in the MQWs. By using different combination of caps, selective area bandgap control is possible.

The deposition of a thin layer of sputtered $\mathrm{SiO} 2$ and a subsequent high temperature annealing is a simple, reliable and low cost technique for QW intermixing. It has been used to intermix a number of different material systems, including GaAs/AlGaAs, GaInP/AlGaInP, InGaAs/InGaAsP, \& InGaAs/InAlGaAs multiple QW structures [5]. The technique allowed large bandgap shifts to be obtained for a range of above material systems, covering the wavelength range $600-1600 \mathrm{~nm}$. Using an appropriate masking, it was possible to completely suppress the intermixing process. It provided an important tool for photonic integration.

The degree of intermixing for an impurity free interdiffusion enhancement technique using the dielectric cap layer depended on several factors, namely encapsulating dielectric material, the deposition conditions, and thermal treatment conditions. The strength of using this technique was that it was free from the influence of the related impurity and the damages of implanted ions. The oxidized AlGaAs as the dielectric cap layer was demonstrated to be useful as a diffusion mask [6]. A thick AlAs cap layer and $\mathrm{AlGaAs}$ interlayer were grown as a capping layer on the top of the $\mathrm{InGaAs} / \mathrm{GaAs} \mathrm{QW}$ structure. It was observed that the $\mathrm{QW}$ interdiffusion rate increased with the $\mathrm{Al}$ composition of the $\mathrm{Al}_{\mathrm{x}} \mathrm{Ga}_{1-\mathrm{x}} \mathrm{As}$ nterlayer until $\mathrm{x}$ reached about 0.5 and then saturate for $\mathrm{x} \geq 0.5$.

\subsection{Anodic-oxide-induced Intermixing}

A novel impurity-free interdiffusion technique, namely anodic-oxide-induced interdiffusion, has been demonstrated [7, 8]. The sample was rinsed in $10 \% \mathrm{HCl}$ and deionized water, and nitrogen blow dried before it was picked up on the backside by vacuum tweezers made by a stainless steel tube as shown in Fig. 6. A portion of the GaAs cap layer of the GaAs/AlGaAs QW structure was anodically oxidized, and it was observed that the oxide enhanced the interdiffusion at high temperatures. Photoluminescence, transmission electron microscopy, and quantum well model were used to understand the effects of intermixing. The results showed that residual water in the oxide served to increase the intermixing, though it was not the prime cause for intermixing. The injection of defects such as group III vacancies or interstitials was considered to be a driving force for the intermixing. A saturation effect in the interdiffusion was observed for prolonged times for quantum wells of certain thickness. The calculated $\mathrm{Al}$ profile was also compared with the one determined from the TEM method. The activation energy is similar to those obtained from $\mathrm{SiO}_{2}$ cap annealed QW structures.

\section{$2.4 \quad$ Laser-assisted Disordering}

Laser induced QW intermixing has been studied for some time, It is a direct write process that can pattern impurity induced layer disordering. This technique employed a highly focused $\mathrm{Ar}^{+}$laser beam. For fabrication of AlGaAs-GaAs DFQW, the laser beam with lasing wavelength of $488 \mathrm{~nm}$ was scanned through the heterostructure sample encapsulated with a $90 \mathrm{~nm}$ layer of $\mathrm{Si}_{-} \mathrm{Si}_{3} \mathrm{~N}_{4}$. Its scan speed could be as high as $85 \mu \mathrm{m} / \mathrm{s}$. The laser beam interaction region would result in a smooth cylindrical section on the micron scale. Annealing was then applied to drive the Si into the as-grown crystal, resulting in a local mixing of the crystal layers. 


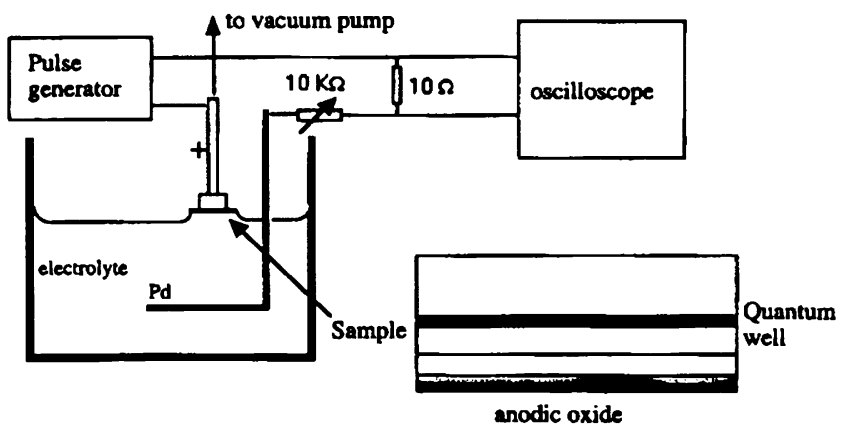

Figure 6 Schematic diagram shows the anodic oxidiztion setup. The electrolyte is composed of ethlene glycol, deionized water, and phosphoric acid (40:20:1 by volume).

Quantum well intermixing in material systems for $1.5 \mu \mathrm{m}$ has been demonstrated using laser disordering techniques and plasma induced damage [10]. The laser disordering required high power densities to melt the material, but the quality of the recrysstallized material might be poo. Besides, if used in high energy, the pulsed mode could introduce thermal shock damage. Photon-absorption induced disordering (PAID) could also be an alternative laser disordering technique. The process relied upon the poor thermal stability of the InGaAsP material system and involved the generation of a high photcreated carrier density through ban-to-band absorption of incident laser photons within the active region of a MQW structure. $100 \mathrm{meV}$ or above blueshift of the photoluminescence peak energy in standard MQW laser structure could be achieved. However, the spatial resolution of PAID, which could be as large as $100 \mu \mathrm{m}$, was limited by the lateral heat flow with using the cw laser. The poor spatial resolution will lead the performance of integrated devices. The spatial resolution has been improved with the technique of pulse photon-absorption induced disordering (P-PAID) [9]. The spatial resolution will improve to below $20 \mu \mathrm{m}$. Recently, the observation of a sub- $\mu \mathrm{m}$ range periodical modulaiton of a band gap caused by gratinglike quantum well intermixing in a intrinsic $\mathrm{GaAs} / \mathrm{AlGaAs} \mathrm{QW}$ structure has been reported. The periodical band gap modulation was caused by the irradiation of the sample with the interference fringe of the second harmonic of a highly coherent Q-switched Nd:YAG laser followed by rapid thermal annealing. This method might be helpful for the fabrication of Distributed Bragg reflector laser, distributed feedback laser and other passive optoelectronics components.

The plasma process induced intermixing has been developed [10]. A new intermixing appeared particularly suitable for long wavelength applications. This technique involved the deposition of a sputtered $\mathrm{SiO}_{2}$ film and subsequent anneals. The technique appeared to be a reliable, low cost and completely suppression of intermixng.

\subsection{Proton Irradiation}

Recently, proton irradiation with subsequent rapid thermal annealing was reported to be selectively inducing layer intermixing and thus shifting the emission wavelengths of $\mathrm{GaAs} / \mathrm{AlGaAs}$ graded-index separate-confinement-heterostructure QW laser [11]. In this structure, up to 40nm shifts were obtained for devices with $1.5 \times 10^{16} \mathrm{~cm}^{-2}$ irradiation dose at temperature around $950^{\circ} \mathrm{C}$ for 30 seconds. A blueshift in the emission wavelength was observed. Using this method, the damage profile for proton was much narrower than that of heavier ions. This could be very promising for integration lasers of different wavelengths for WDM applications.

\section{DIFFUSION MECHANISM}

Recently, the computer simulations of double crystal x-ray diffraction (DCXRD) curves for annealed multiple QW structure, in conjunction with the corresponding experimental data, has been shown to be providing an accurate measure of the diffusion constant in group IV, III-V \& II-VI semiconductors [13]. Not only can simulations of DCXRD rocking curves be 
utilised to quantify the diffusion occurring in post-growth annealing, but they can also be used to determine the amount of diffusion occurring during growth of semiconductor heterostructures. The results showed that the rocking curves of single QW were not sensitive to diffusion. However, the charateractistic of superlattice rocking curves was strongly dependent upon diffusion. It was proposed that this technique could be used to measure the diffusion coefficient.

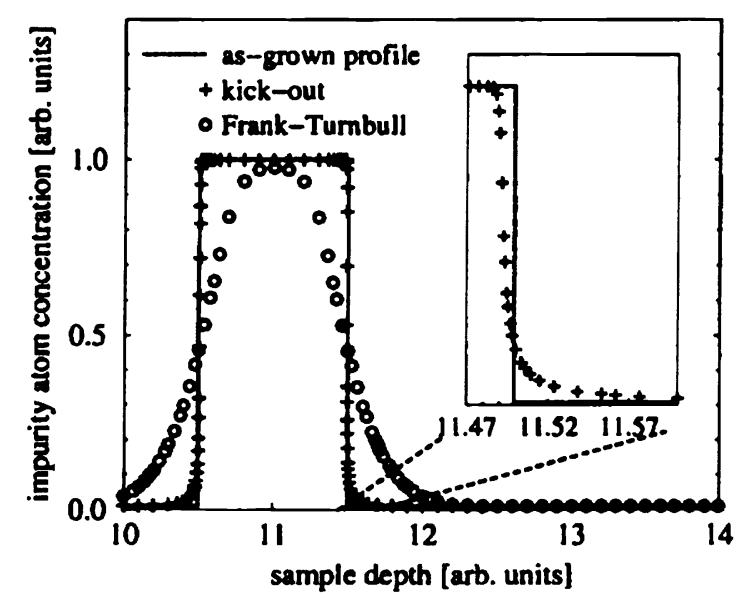

Figure 7. Simulated out-diffusion profiles for the kick-out mechanism and the Frank-Trunbull mechanism under nonequilibrium conditions of intrinsic point defects. Both simulations were performed assuming identical point defect concentrations and diffusivities. The insert shows a magnification of the kick-out profile demonstrating the asymmetric shape of the profile in contrast to the symmetric profiles expected for the Frank-Turnbull mechanism.

Intermixing experimentals with $\mathrm{GaAsP} / \mathrm{GaAs}$ and $\mathrm{GaAsSb} / \mathrm{GaAs}$ superlattice samples were reported at various temperatures and arsenic vapour pressures [12]. The superlattices were grown with mixed crystal (GaAs, GaP, and GaSb) layers containing low $\mathrm{P}$ and $\mathrm{Sb}$ concentrations between 2 and $20 \%$ of the group-V atomic content by MOCVD. The SIMS profiles of the annealed samples showed almost homogeneous interdiffusion, which could be described by constant effective diffusion coefficients. The experimental results of diffusion coefficients show indicated significant influence of lattice strain or material composition on the diffusion properties. Results show that only the kick-out or interstitialcy mechanism see Fig. 7, can explain the interdiffusion experiments. It was also concluded that the interdiffusion process in the superlattice sample is governed by a substitutional-interstitial diffusion mechanism.

\section{OPTICAL PROPERTIES}

\subsection{Optical Gain}

A theoretical analysis on the effect of interdiffusion on the optical gain, linewidth enhancement factor, and the injection current density of $\mathrm{InGaAs} / \mathrm{GaAs}$ and $\mathrm{AlGaAs} / \mathrm{GaAs} \mathrm{QW}$ lasers has been reported [14]. The electron and hole subband structure has been calculated taking into account the effects of valence band mixing and strains. The optical results showed that the gain spectrum could be blue-shifted without an enormous increase in the injected current density. After imposing an upper limit (416 $\mathrm{A} \mathrm{cm}^{-2}$ ) on the injected current density of a typical laser structure, it was found that the InGaAs/GaAs and AlGaAs/GaAs QW lasers could be blue-shifted by 24 and $54 \mathrm{~nm}$ respectively. These predictions could well be compared to the tuning ranges of 53 and $66 \mathrm{meV}$ found for $\mathrm{AlGaAs} / \mathrm{GaAs} \mathrm{QW}$ 's in some experiments. This indicated that the interdiffusion technique could be useful for the tuning of laser operation wavelength for multiwavelength applications. 
For the multiple cations interdiffusion in InGaAs/InAlAs QW, the interdiffusion process could be modelled by the expanded form of Fick's Law. The interdiffusion of In could result in a maximum compressive strain of $0.64 \%$ when annealing time reached $3 \mathrm{hrs}$ at $812^{\circ} \mathrm{C}$. For small interdiffusion (i.e. 1-1.5hrs), the subband separation between the lowest heavy and light hole was the greatest, which results in an enhanced optical gain up to $40 \%$, modified band structure and DOS. Besides, this provides the best lasing performance. For large interdiffusion (i.e. $6 \mathrm{hrs}$ ), a large blue-shifted of peak gain from 0.842 to $1.016 \mathrm{eV}$ (1.47 to $1.22 \mu \mathrm{m})$ was reported [15], thus providing a high tunability of lasing wavelength.
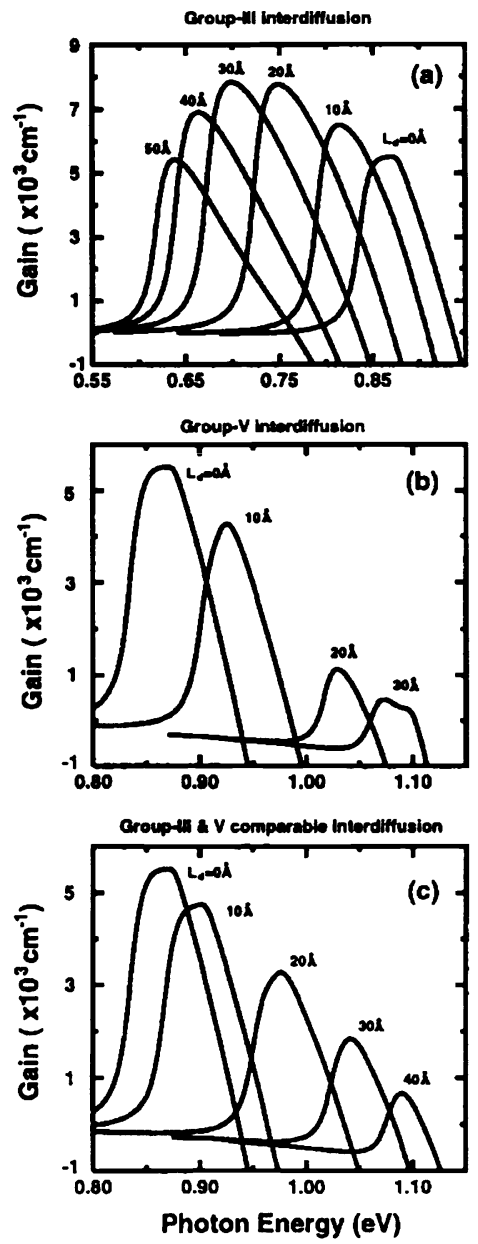

Figure 8 The gain spectra of $\operatorname{In}_{0.53} \mathrm{Ga}_{0.47} \mathrm{As} / \mathrm{InP} \mathrm{QW}$ with asgrown well width of $60 \AA$ and at a fixed carrier injection level of $5 \times 10^{12} \mathrm{~cm}^{-2}$ for various diffusion length with three type of interdiffusion: (a) group-III interdiffusion, (b) group-V interdiffusion, and (c) group (III\&V) interdiffusion.

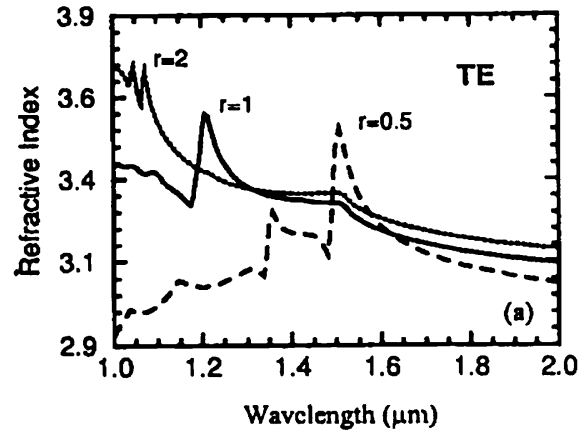

(a)

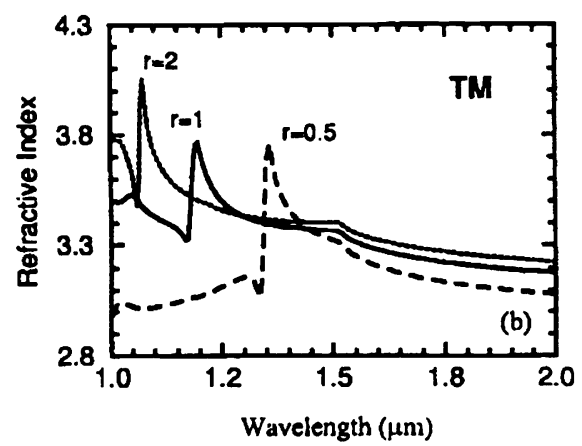

(b)

A theoretical analysis on the effect of interdiffusion in InGaAs/InP reported that [16] an enhancement of the material gain by $42 \%$ could be obtained by the group III interdiffusion at a diffusion length of $30 \mathrm{~A}$. A large red shift of $240 \mathrm{meV}$ of the peak gain from 0.87 to $0.63 \mathrm{eV}$ has been reported [16]. The optical gains of $\mathrm{In}_{0.53} \mathrm{Ga}_{0.47} \mathrm{As} / \mathrm{InP} \mathrm{QW}$ for group-III, group-V, and comparable group (III \& V) interdiffusion are shown in Fig. 8 (a)-(c) respectively. The sheet carrier density used in the 
calculation is $5 \times 10^{12} \mathrm{~cm}^{-2}$. For QW lasers, the biaxial compressive strain in the well could reduce the valence subbands mixing and improve their parabolicity, resulting in a small effective mass. The advantage of a small effective mass was that it could reduce the threshold carrier density and the lower threshold carrier density. This effect was expected not only to reduce the threshold current but also to improve other lasing characteristic.

\subsection{Absorption and Refractive Index}

A comprehensive model [17] has been developed for the calculation of polarisation-dependent absorption and refractive index of InGaAs-InP interdiffused multiple-quantum-well at room temperature for wavelengths ranging from 1.1 to $2.4 \mu \mathrm{m}$. Group III and V types of interdiffusion are considered separatly. The as-grown structure was a latticed-matched $\mathrm{In}_{0.53} \mathrm{Ga}_{0.47} \mathrm{As}$-InP structure with a well width of $60 \AA$. The optical transitions consist of a full quantum well calculation together with $\Gamma, \mathrm{X}$, and $\mathrm{L}$ valleys contributions. The Kramers-Krönig transformation was used to link the real and imaginary parts of the dielectric functions. The results showed that group-III only interdiffusion produced compressive strain and resulted in a bandedge red shift and refractive index enlargement, while the tensile strain induced by group- $\mathrm{V}$ only interdiffusion resulted in a vice versa effect, as shown in Fig. 9 (a)-(b). This provided a left and right tunable band edge and positive and negative index steps depending on the interdiffusion process. A small and constant briefringence of 0.005 at around $1.55 \mu \mathrm{m}$ could also be obtained over a $50 \mathrm{~nm}$ wavelength range using group- $\mathrm{V}$ only interdiffusion. These properties had strong implications for the realising of a tunable and high performance device as well as for photonic integrations.

\section{DEVICES}

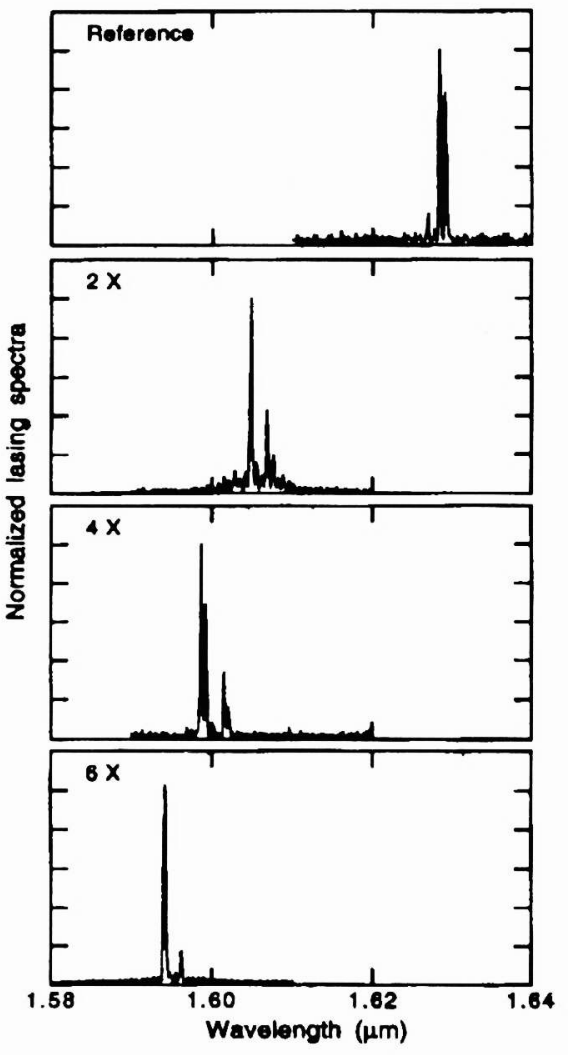

Figure 10 Normalized emission spectra of the broad area lasers at room temperature. The top graph corresponds to an unimplanted sample.

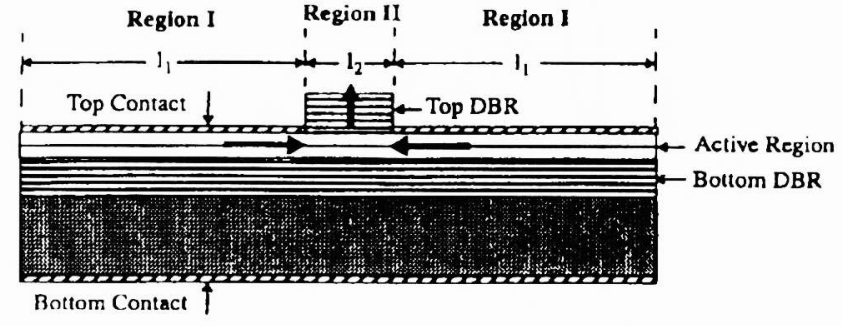

Figure 11 Schematic of Hybrid-Caavity Surface Emitting Laser

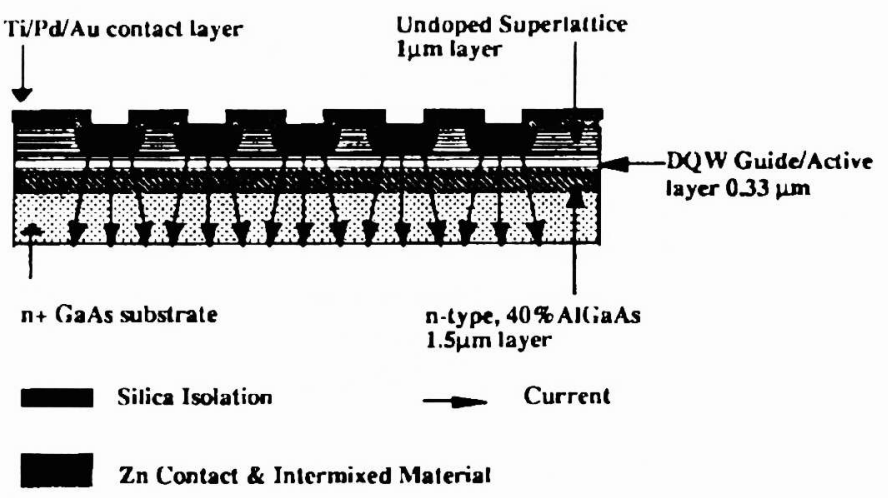

Figure 12. Structure of antiguided array laser 
An InGaAsP/InP laser diodes with blue shifted emission wavelength has been realized with a quantum well interdiffusion process based on low-energy ion implantation [18]. The method was to generate vacancies near the surface of an incomplete laser structure, for which the epitaxial growth was interrupted $45 \mathrm{~nm}$ above the active layers of the devices, using low-energy implantation $\left(18 \mathrm{keV} \mathrm{As}^{+}\right)$. The vacancies then underwent diffusion through the quantum wells by rapid thermal annealer at the temperature of $685^{\circ} \mathrm{C}$. The interdiffusion caused the atoms at the interfaces of the active layers to intermix locally and thus resulting in an increase of the band gap energy. In order to obtain a large blueshifts, multiple processing cycles of implantation at low dose and low energy were conducted followed by a thermal annealing. The normalised spectra of the laser were shown in Fig. 10. The four emission spectra represent the laser fabricated from the unimplanted region, two, four, and six times implantation. It can be found that the lasing threshold for the implanted samples was about 2.5 times higher than that of the unimplanted samples but the former appeared to be independent of the number of processing cycles. In this structure which was based on the InGaAsP quaternary system, blueshifts as large as $35 \mathrm{~nm}$ were obtained.

Vertical-cavity surface-emitting laser (VCSEL) has been analyzed to show the possibility of optical pumping from an integrated edge-emitting laser [19]. It can be employed to develop VCSEL's of ultra small dimensions or closely spaced VCSEL arrays. In such a hybrid-cavity surface emitting laser (HCSEL). As shown in Fig. 11, normal current injection of the edgeemitting device produces the edge-emitting or in-plane mode. In this figure, region I acts as an edge-emitting electrically injected laser and region II as a VCSEL that is pumped optically. The laser design utilizes dopant induced intermixing of quantum wells to provide different gain regions for the surface and edge-emitting sections. It has been shown that it is possible for the efficient surface photon emission to occur at high vertical-cavity mirror reflectivities and when sufficient QW bandgap separation energy is provided.

The fabrication of an antiguided laser array using zinc diffusion induced intermixing of a superlattice has been studied in order to create the necessary index step without the need for overgrowth [20]. The fabrication made use of five-element, 10$\mu \mathrm{m}$ center, antiguided array lasers (Fig. 12) in the GaAs-AlGaAs system operating at $0.860 \mu \mathrm{m}$, with the device being operated at $1.2 \mathrm{x}$ diffraction limit to $3-\mathrm{W}$ pulsed (total, both facets) and 1.6-W quasi-continuous-wave (CW) (100- $\mu$ s pulses; total, both facets). After the intermixing of GaAs-AlAs superlattices, the refractive index changed within the range 4.8\%-5.2\%, making it sufficient to create an effective index step, $\Delta \mathrm{n}_{\mathrm{eff}} \approx 0.015$ between the antiguide and interelement regions of the array.

The generation mechanism of self-sustained pulsation in vertical-cavity surface-emitting lasers (VCSEL's) was analysed with the influence of lateral loss effects, such as self-focusing, lateral diffraction loss, and spatial hole burning, taken into consideration [21]. It was shown that the lateral loss effects had significant influence on the excitation of self-sustained pulsation (SSP), especially for VSCEL's with small size (i.e. $\mathrm{W}<1 \mu \mathrm{m}$ ). A diffused quantum-well (DFQW) structure for VCSEL's was proposed to enhance SSP. It has been shown that such structure enhances the lateral overlap effects but minimizes the influence of lateral loss effects due to the maximisation of the overlap volume between the absorption region, lasing mode and the core radius of the VCSEL. In addition, peak power and high repetition frequency can also be improved. It is believed that the DFQW structure can also be applied to facet-emitted lasers for the enhancement of SSP.

\subsection{Electro-optic modulators}

The III-V semiconductor QW electrooptical phase modulators are of interest for a range of applications in optical communication and signal processing due to their large electrooptic effect. Waveguide phase modulators, with 0.5- and 1- $\mu \mathrm{m}$ QW active regions defined by impurity induced disordering were reported [25]. By controlling the extent of the interdiffusion in the lateral claddings, the refractive index difference between the core and cladding was used to provide a single-mode operation. The structure of the phase modulator is shown in Fig. 13. Strong optical confinement required the peak impurity concentration to be at the centre of the QW active region. Moreover, the annealing time needed to be optimized so that single mode could be maintained at the desired bias field. A low dopent concentration was also expected to minimize the destruction of the modulator structure. The results showed that since the core/cladding interface was graded, the width of the metal contact 
would be important. A comparison of modulation efficiency for active layer thickness of 0.5 and $1.0 \mu \mathrm{m}$ showed that the $0.5-\mu \mathrm{m}$ one is a more efficient structure and its absorption loss could be reduced by increasing the applied field 50 to $100 \mathrm{kV} / \mathrm{cm}$.

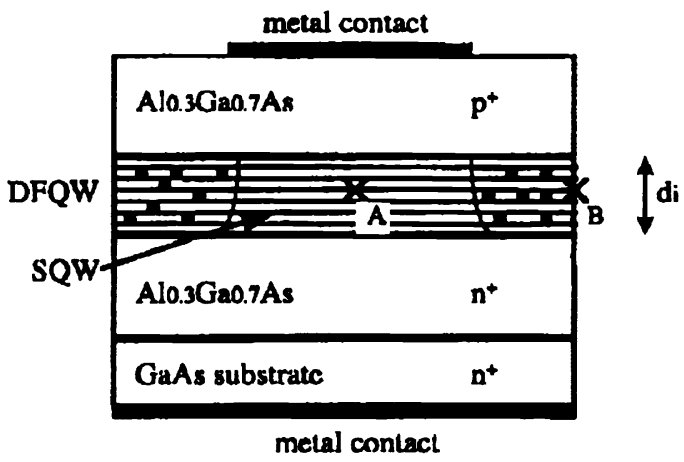

Figure 13 The cross section of the waveguide-type phase modulator, where the guiding region contains square QW's after interdiffusion.

Polarization-insensitive electroabsorptive III-V semiconductor QW modulation devices have been under intensive development because they can accommodate randomly polarized optical signals. A paraboliclike QW structure can be used to produce polarization-insensitive waveguide type modulators [26]. The use of interdiffusion of $\mathrm{AlGaAs} / \mathrm{GaAs} \mathrm{QW}$ to achieve the parabolic well shape was proposed. The theoretical results indicated that interdiffused $\mathrm{QW}$ could produce equal eigenstate spacing polarization insensitive Stark shifts, and modulation similar to an ideal parabolic quantum well. Three procedures were also proposed to develop polarization- insensitive on and off states in paraboliclike interdiffused QW. The modulation depth was compatible with that of the measured parabolic QWs. For diffused QWs one could take advantage of an as-grown rectangular QW with postgrowth thermal processing. These features demonstrate that an interdiffused QW structure could be used to produce a polarisation-insensitive electroabsorption modulator.

The QW electro-absorption (EA) modulator has been regarded recently as an important technique in the development of high speed optical communication systems. The effect of $\mathrm{Al}$ concentration in barrier and the thickness of the well layer on the modulation properties of $\mathrm{AlGaAs} / \mathrm{GaAs} \mathrm{QWs}$ was theoretically studied [28]. The results showed that, with a larger $\mathrm{Al}$ concentration and a wider well depth, the range of interdiffusion for an enhanced electro-absorption change increased with both of these parameters, while insetion loss increased with the former and decreased with the latter. However, the increase in loss was lower than that of the rectangular $\mathrm{QW}$ for the same magnitude of absorption change. The range of a tunable absorption-peak wavelength produced by interdiffusion increased with increasing $\mathrm{Al}$ concentration and decreased with increasing well width. Moreover, in a moderately interdiffused QW, the required bias was reduced for the same level of EA modulation. For the best device operation, interdiffused QWs with the $\mathrm{Al}$ concentration between 0.3 and 0.4 and well width between 10 and $12 \mathrm{~nm}$ were the most suitable for developing a general-purpose electro-absorptive modulator. When applied in high-speed modulators, the EA of a wide and shallow QW active-region structure can be further enhanced with the use of corresponding interdiffusion.

\subsection{Vertical Cavity Waveguides}

During the past few years, vertical cavity devices using semiconductor multiple QW have given rise to a variety of novel devices. The use of vertical cavity is of great interest because of their high packing density, low threshold current, and their suitability for parallel free-space optical interconnect. The technique of impurity induced disordering of the QW composition between layers has recently been used to investigate $\mathrm{AlGaAs} / \mathrm{GaAs} \mathrm{QW}$ vertical cavity waveguides [23]. The model was based on a two dimensional description profile which would produce QW intermixing. In a long cavity, guided mode could be supported by using higher implantation energy, but a larger mask diameter should be used to maintain strong guiding. Results 
showed that optical lateral confinement had been accomplished, see Fig. 14, with more than $70 \%$ of the power gathered in the cavity. The fraction of power confined in the waveguide was shown to be improved by $30 \%$ after annealing. In addition, waveguide loss was estimated to be less than $40 \mathrm{~cm}^{-1}$ which resulted in less than $1 \mathrm{~dB}$ for the structures.

\section{LP 01 mode along vertical direction}

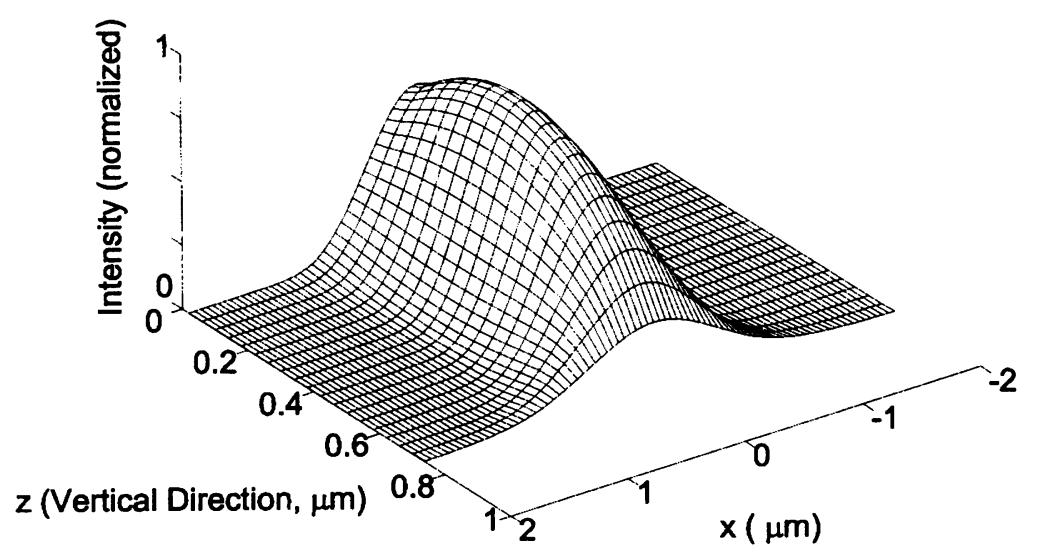

Figure 14. Mode intensity profile for waveguide with implant energy $=600 \mathrm{keV}$, mask radius $=1 \mu \mathrm{m}$; annealed for $91 \mathrm{~s}$ and $\lambda=852 \mathrm{~nm}$ in 3 -dimensional profile.

\subsection{Monolithic Integration}

Photonic integrated circuits (PIC's) is of considerable interest for monolithic integration of active and passive optical components. The realization of PIC would be required to control the local optical and electrical characteristics across a wafer. QW intermixing is a promising approach to selectively modify the QW structure. The use of a laser irradiation process, which combines irradiation by continuous wave and Q-switched pulsed Nd:YAG lasers, were simultaneously employed to induce QW intermixing in an InGaAs-InGaAsP structure [24]. Extended cavity-ridge lasers with $800-\mu \mathrm{m}$-long active sections and $1000 \mu \mathrm{m}$ long passive sections were fabricated. Differential shifts up to $70 \mathrm{meV}$ have been obtained. The intermixed single-mode waveguide loss was $2.1 \mathrm{~cm}^{-1}$ at $1556 \mathrm{~nm}$. The slope efficiency of the extended cavity lasers was very close to that of $800 \mu \mathrm{m}$ long all active lasers, and the threshold current was $10 \mathrm{~mA}$ higher that for an $800-\mu \mathrm{m}$-long all-active device.

\section{CONCLUSION}

The success of using QW's structures to fabricate high performance photonic devices depends upon whether the intermixing technique is compatible with the existing fabrication technique. This $\mathrm{QW}$ intermixing technique has been developed with a selective area means, such as ion implantation and impurity-free vacancy diffusion. It has been recently found out that anodic-oxide-induced intermixing, laser-assisted disordering and proton irradiation are also developed for realization of interdiffused QW structure. It has been shown that QWI can improve the optical properties. These developments have strong implications for the realization of a tunable and high performance device such as laser, electro-optic modulator, vertical cavity waveguide and their monolithic integration for building Photonics IC's. 


\section{ACKNOWLEDGEMENTS}

The author would like to thank the Research Grant Council (RCG) earmarked grant of Hong Kong and the University of Hong Kong CRCG grant.

\section{REFERENCES}

[1] E.H. Li, Editor, Quantum Well Mixing and Optoelectronic Device Applications, Milestone Series, SPIE Press, Bellingham (1998) vol. 145.

[2] B. S. Ooi, Y. S. Tang, A. Saher Helmy, A. C. Bryce, J. H. Marsh, M. Paquette, J. Beauvais, J. Appl. Phys. 83, 4526 (1998).

[3] J.E. Hayson, P.J. Poole, Y. Feng, E.S. Koteles, J.J. He, S. Charbonneau, R.D. Goldberg, I.V. Mitchell, J.Vac.Sci.Technol. A 16, 817 (1998).

[4] P.G. Piva, R.D. Goldberg, I.V. Mitchell, Huajie Chen, R.M. Feenstra, G.C. Weatherly, D.W. McComb, G.C. Aers, P.J. Poole, S. Charbonneau, Appl. Phys. Lett. 72, 1599 (1998).

[5] O.P. Kowalski, C.J. Hamilton, S.D. McDougall, J.H. Marsh, A.C. Bryce, R.M. De La Rue, B. Vögele, C.R. Stanley, Appl. Phys. Lett. 72, 581 (1998).

[6] J.-S. Choe, S.-W. Ryu, B.-D. Choe, H. Lim, J. Appl. Phys. 83, 5779 (1998).

[7] S. Yuan, Y. Kim, H.H. Tan, C. Jagadish, P.T. Burke, L.V. Dao, M. Gal, M.C.Y. Chan, E.H. Li, J. Zou, D.Q. Cai, D.J.H. Cockayne, R.M. Cohen, J. Appl. Phys. 83, 1305 (1998).

[8] S. Yuan, C. Jagadish, Yong Kim, Y. Chang, H.H. Tan, R.M. Cohen, M. Petravic, L.V. Dao, M. Gal, M.C.Y. Chan, E.H. Li, J.S.O, P. S. Zory, IEEE J. Sel. Top. Quantum Electron., 1998. (accepted for publication)

[9] J.-J. Shin, S. Gurtler, Y. Chang, C.C. Yang, Appl. Phys. Lett. 72, 2808 (1998).

[10] J.H. Marsh, O.P. Kowalski, S.D. McDougall, B.C. Qiu, A. McKee, C.J. Hamilton, R.M. De La Rue, A.C. Bryce, J. Vac. Sci. Technol. A 16, 810 (1998).

[11] H.H. Tan, C. Jagadish, Appl. Phys. Lett. 71, 2680 (1997).

[12] M. Schultz, U. Egger, R. Scholz, O. Breitenstein, U. Gösele, T.Y. Tan, J. Appl. Phys. 83, 5295 (1998).

[13] J.M. Fatah, P. Harrison, T. Stirner, J.H.C. Hogg, W.E. Hagston, J. Appl. Phys. 83, 4037 (1998).

[14] K.S. Chan, E. Herbert Li, M.C.Y. Chan, IEEE J. Quantum Electron. 34, 157 (1998).

[15] M.C.Y. Chan, Y. Chan, E. Herbert Li, IEEE J. Quantum Electron. 34, 519 (1998).

[16] M.C.Y. Chan, E. Herbert Li, and K.S. Chan, Physica B 245, 317 (1998)

[17] E. H. Li, IEEE J. Quantum Electron. 34, 982 (1998).

[18] M. Paquette, J. Beauvais, J. Beerens, P. J. Poole, S. Charbonneau, C. J. Miner, and Blaauw, Appl. Phys. Lett. 71, 3749 (1998).

[19] M. D. Gerhold, A. I. Onischenko, J. Sarma, and P. Bhattacharya, IEEE J. Quantum Electron. 34, 506 (1998).

[20] J. M. Gray, J. H. Marsh, and J. S. Roberts, IEEE Photon. Technol. Lett. 10, 328 (1998).

[21] S. F. Yu, IEEE J. Quantum Electron. 34, 497 (1998).

[22] S. F. Yu and E, H. Li, IEEE J. Select. Topics Quantum Electron. 1998. (accepted for publication)

[23] A. T. H. Li, C. C. Chan, and E. H. Li, IEEE J. Lightwave Technol., 1998. (accepted for publication)

[24] B. C. Qiu, A. C. Bryce, R. M. De La Rue, and J. H. Marsh, IEEE Photon. Technol. Lett. 10, 769 (1998).

[25] W. C. H. Choy ,B. L. Weiss, and E. H. Li, IEEE J. Quantum Electron. 34, 84 (1998).

[26] W. C. H. Choy and E. H. Li, Applied Optics 37, 1674 (1998).

[27] E. H. Li, IEEE J. Quantum Electron. 1998. (accepted for publication)

[28] W. C. H. Choy and E. H. Li, IEEE J. Quantum Electron. 1998. (accepted for publication) 